\title{
Noise-induced enhancement of stability in a metastable system with damping
}

\author{
Alessandro Fiasconaro, ${ }^{1,2,3, *}$ Juan J. Mazo, ${ }^{1,4}$ and Bernardo Spagnolo ${ }^{3}$ \\ ${ }^{1}$ Departamento de Física de la Materia Condensada, Universidad de Zaragoza, E-50009 Zaragoza, Spain \\ ${ }^{2}$ Centro Universitario de la Defensa de Zaragoza, Ctra. de Huesca s/n, E-50090 Zaragoza, Spain \\ ${ }^{3}$ Dipartimento di Fisica e Tecnologie Relative, Group of Interdisciplinary Physics, Università di Palermo and CNISM-INFM, \\ Viale delle Scienze, I-90128 Palermo, Italy \\ ${ }^{4}$ Instituto de Ciencia de Materiales de Aragón, CSIC-Universidad de Zaragoza, E-50009 Zaragoza, Spain
}

(Received 26 April 2010; revised manuscript received 10 September 2010; published 22 October 2010)

\begin{abstract}
The mean first passage time of a Brownian particle from an initial unstable state in a metastable system with damping is investigated. The system is analyzed in the low to high damping regime, and the role played by the damping parameter is studied. We observe the noise enhanced stability effect for all the initial unstable states under study and for all values of the damping parameter $\gamma$ investigated. The curves show a behavior of the mean first passage time vs $\gamma$ very close to that observed for an overdamped particle in the presence of colored noise as a function of the correlation time.
\end{abstract}

DOI: 10.1103/PhysRevE.82.041120

PACS number(s): 05.40.-a, 02.50.-r

\section{INTRODUCTION}

The study of nonlinear dynamical systems in the presence of external noise has led to the discovery of a number of counterintuitive phenomena, with a constructive role of the noise and high fundamental and practical interests in many scientific areas.

One of these effects is the noise enhanced stability (NES). This effect is observed in potential profiles with metastable states. The system stays close to the potential well for a larger time than in the deterministic case (dynamical time) and the average residence time shows either a nonmonotonic or divergent behavior. The observed nonmonotonic resonancelike behavior proves to be different from the monotonic one of the Kramers theory and its extensions [1,2]. This enhancement of stability, first noted by Hirsch et al. [3], has been observed in different physical and biological systems [4-12]. Specifically, this effect belongs to a highly topical interdisciplinary realm of studies, ranging from condensedmatter physics to molecular biology or to cancer growth dynamics. Recently, the NES effect has been studied in fluctuating bistable potentials [13], ecological systems [14], timedelayed metastable systems [15], magnetic systems [16], overdamped and underdamped Josephson junctions [17], and experimentally detected in an oscillator chemical system (the Belousov-Zhabotinsky reaction) [18] and in underdamped Josephson junctions [19].

When considering a Brownian particle moving in a metastable fluctuating potential, the NES effect is always observed, regardless of the unstable initial position of the particle [6]. However, from a theoretical point of view, the study of the role of different unstable initial conditions in nonfluctuating potentials, as the one studied here, provides a better understanding of the occurrence of the NES phenomenon. As shown in $[6,20]$, depending on the initial conditions, two different dynamical regimes can occur: one is characterized by a nonmonotonic behavior of the average escape time as a

\footnotetext{
*afiascon@unizar.es
}

function of noise intensity and the other features a divergence of the mean escape time when the noise intensity tends to zero, implying that the Brownian particle remains trapped within the potential well in the limit of small noise intensities.

In static potentials, the NES is characterized by mean first passage time (MFPT) values greater than deterministic ones for the Brownian particle which reach a given far boundary starting from an initial unstable position beyond the potential well $[6,20]$. For any of these unstable initial positions it is possible to define a "NES range" given by the interval of noise intensity values for which the NES effect is observed $[5,6]$. Concerning the initial unstable conditions, the physical system can be brought in this nonequilibrium unstable state by a sudden change of control parameters. Examples of such situations include spinodal decomposition in the dynamics of phase transitions and the process of laser switch-on (see Refs. $[4,13]$ in [6]). To complement the theoretical analysis of transient dynamics in metastable systems we introduced in [20] a new signature of the NES effect: the presence of a minimum in the behavior of a generalization of the Lyapunov exponent for stochastic systems as a function of the noise intensity. Of course this behavior depends on the initial conditions. From experimental point of view, as far as we know, the majority of the observations of the NES effect concerns experiments with fluctuating barriers and with initial conditions in the metastable state. However, it may be possible to put the experimental setup in an initial unstable nonequilibrium condition (see Ref. [6]). In a recent experimental work on the Belousov-Zhabotinsky reaction [18] it has been observed a noise-induced stabilization characterized by a decrease of the maximum Lyapunov exponent (see Ref. [20]) and an increase of the passage time of the laminar region (see Ref. [3]). The dynamics of this chemical reaction, in fact, can be described by a Langevin equation with a fixed metastable potential profile.

The present paper is a continuation of the work in [20,21], where noise-induced enhancement of stability in a metastable potential in the overdamped limit was studied. There it is shown a clear divergent behavior of the MFPT as a function of the noise intensity at some initial positions $x_{0}$, 


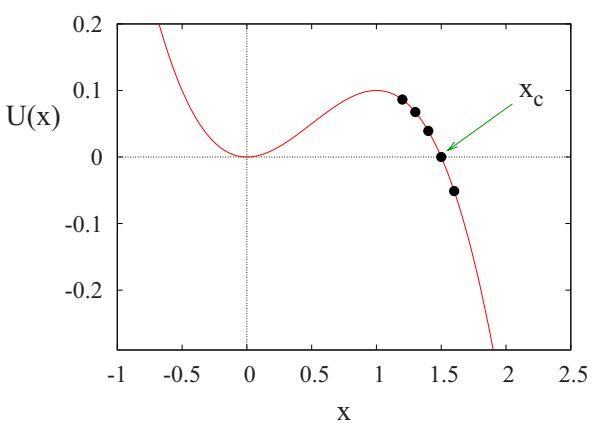

FIG. 1. (Color online) The cubic potential $U(x)=a x^{2}-b x^{3}$ with some of the initial positions investigated (dots), namely, $x_{0}$ $=1.2,1.3,1.4,1.5,1.6$. The position $x_{c}=1.5$ represents, for the overdamped motion in the presence of white noise [see Eq. (2)], the critical initial position which separates the set of the initial unstable states producing divergency for $D \rightarrow 0$ from those which give only a nonmonotonic behavior of the average escape time $[6,8,20,21]$.

namely, for $x_{\max }<x_{0}<x_{c}$ [with $x_{\max }$ as the position of the potential maximum and $x_{c}$ as the position where the potential shape intersects the $x$ axis (see Fig. 1)]. This behavior had been already reported in $[6,8]$. The results are presented as a signature of noise enhanced stability effect. This approach allowed for the characterization of the stochastic equilibrium position [20] and motivated the investigation on how the realistic noise sources modify the escape process [21]. The purpose of our study is to introduce damping in the system and extend the results presented in [20]. In particular, we are interested in how the damping affects the divergent behavior there shown and how the critical point $x_{c}$ depends on the damping.

Specifically, by decreasing the value of the damping parameter, we observe the following: (i) a shift of the increase of the mean first passage times (NES effect) toward higher noise intensities, (ii) a decreasing of the value of the MFPT maximum; (iii) a decrease of the critical initial position, $x_{c}^{*}$, which separates the set of the initial unstable states giving rise to divergence of the MFPT for noise intensity $D \rightarrow 0$ $[6,8,20,21]$ from those which give nonmonotonic behavior with a maximum of MFPT; and (iv) a trend to the disappearance of the divergent dynamical regime for given initial position.

These peculiarities of the behavior of the MFPT appear similar to those observed in the study on the correlation time of the Gaussian colored noise for an overdamped Brownian particle moving in a metastable potential [21]. In particular, by decreasing the damping parameter in our system, the behavior of the MFPT from the initial unstable state as a function of the white-noise intensity is quite similar to that obtained by increasing the correlation time $\tau_{c}$ of the colored noise in the overdamped case.

\section{MODEL AND RESULTS}

The starting point of this study is the Langevin equation of motion,

$$
\ddot{x}+\gamma \dot{x}=-\frac{d U(x)}{d x}+\xi(t),
$$

where $\xi(t)$ is the white Gaussian noise with the usual statistical properties: $\langle\xi(t)\rangle=0$ and $\left\langle\xi(t) \xi\left(t^{\prime}\right)\right\rangle=2 \gamma D \delta\left(t^{\prime}-t\right)$, where
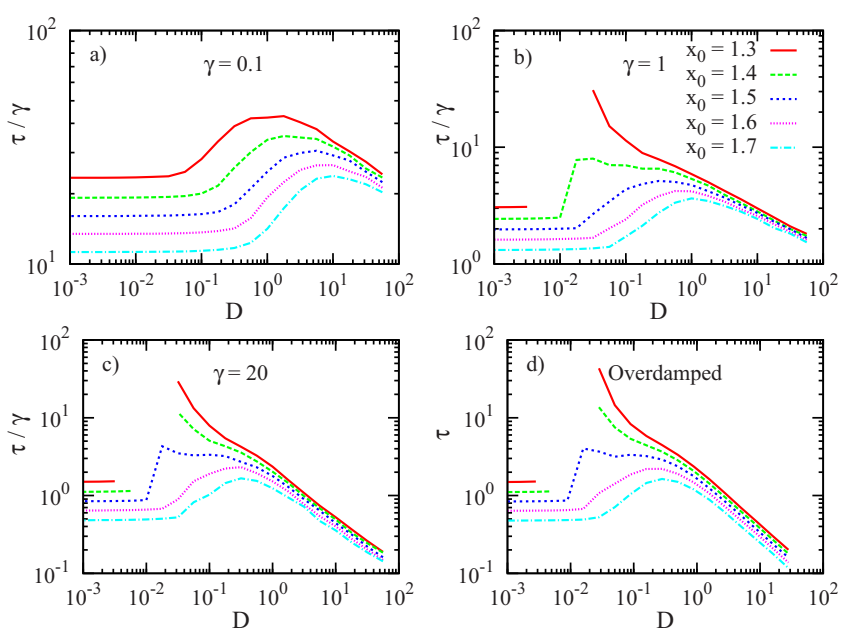

FIG. 2. (Color online) Log-log plots of the mean first passage time $\tau$ as a function of $D$ for different values of the damping, namely, $\gamma=0.1,1,20$, and for the initial positions $x_{0}$ $=1.3,1.4,1.5,1.6,1.7$. The panels $(\mathrm{a})-(\mathrm{c})$ show the values rescaled in time to allow the comparison with the overdamped case [panel (d)] obtained by numerical simulation in Eq. (2).

$\gamma D$ gives the noise intensity with $D$ as a dimensionless temperature.

The potential $U(x)$ used in Eq. (1) is $U(x)=a x^{2}-b x^{3}$, with $a=0.3$ and $b=0.2$. The profile has a local stable state at $x$ $=0$ and an unstable equilibrium state at $x=1$ (see Fig. 1). We are interested in computing the average escape time of the system starting from an unstable position beyond the potential well. The evaluation of the MFPT values, $\tau$, as a function of the noise intensity has been performed by averaging over $N=20000$ realizations the times $t_{i}$ spent by the particle to reach the exit boundary $x_{f}$ in each trajectory calculated from the stochastic differential equation [Eq. (1)], $\tau=1 / N \sum t_{i}$. The absorbing boundary for the escape process is set at $x_{F}=2.2$ and the maximum simulation time at $t_{\max }=20,000$ in arbitrary units. We choose to plot the results when all the realizations reach the absorbing boundary within the maximum simulation time, and, consequently, some of the shown curves are interrupted at some $D$ values. This means that the shown MFPT values are calculated with the complete statistics. The chosen values of $t_{\max }$ and $N$ are large enough that they do not affect the shape of the plotted curves but only the maximum $\tau$ values that are possible to calculate without ambiguity in the divergent behavior cases. For all the initial unstable states (some of them visible in Fig. 1) and all the damping parameters considered we find an increase of the MFPT $\tau$ with respect to the deterministic values.

Figure 2 shows the calculations of $\tau$ vs $D$ performed with different damping parameters $(\gamma=0.1,1,20)$ and a comparison with the overdamped case [panel (d)] obtained by numerical simulation of Eq. (2). We see that, as expected at high damping, the mean first passage time $\tau$, rescaled by a factor $\gamma$, reproduces the overdamped behavior studied in [20] by integrating the corresponding Langevin equation 


$$
\dot{x}=-\frac{d U(x)}{d x}+\xi(t),
$$

where $\xi(t)$ is a white $\delta$-correlated Gaussian noise with zero mean and the time $t$ is here rescaled as $t / \gamma \rightarrow t$. For very small $D$, the noise barely affects the dynamics and most of the particles escape approximately at the deterministic escape time, except a few ones which remain trapped into the potential well and cause the divergence. This seems to contradict the constant behavior of the MFPT for $D \rightarrow 0$, as shown in Fig. 2. This is due to the fact that the probability that some particle is temporary trapped is very low and strongly decreases with the noise intensity. Therefore, because of this, we do not observe such particles in simulations and the average escape time becomes equal to the deterministic time with a flat behavior of MFPT for $D \rightarrow 0$ (see Ref. [8] for details).

Due to the limited number of particles in computer simulations, all curves of MFPT as a function of $D$ in Figs. 2 and 4 , at very low noise intensities, show the same flat behavior. If we increase the noise, we reach a point where in the simulations at least one realization moves backward and the Brownian particle enters the potential well. Depending on the value of the unstable initial position and damping, it is possible then that this particle remains into the well for all our observation time. This is the case of small $x_{0}$ and high damping [see, for instance, the curves for $x_{0}=1.3$ or 1.4 in Fig. 2(c)]. Increasing the noise we reach a value high enough to guarantee the observation of the thermal escape of the particle out of the well, after entering in it. Beyond this point, a monotonous decreasing of the mean passage time is observed. However, at larger values of $x_{0}$ or smaller damping all the particles reach the adsorbing boundary within our observation time.

As seen in all panels of Fig. 2, the $\tau$ versus $D$ curves show either a nonmonotonic behavior with a well visible hump at moderate values of the noise intensity or a divergent behavior. Both behaviors are a signature of the NES effect, which is understood as an increase of the mean first passage time with respect to the dynamical time $[6,20]$.

Figure 3 complements our information on the dynamics of the system given in Fig. 2. There we plot the standard deviation $\sigma$ of the passage time probability distribution [Figs. $3(\mathrm{a})-3(\mathrm{c})]\left(\sigma^{2}=1 / N \Sigma t_{i}^{2}-\tau^{2}\right)$. For high values of the damping parameter, we notice a huge increase of the $\sigma$ for low values of noise intensity, demonstrating a strong enlargement of the distribution when the particle feels a noise intensity comparable with the height of potential barrier. As a general remark, $\sigma$ follows the same trend as $\tau$ : divergent behavior when $\tau$ diverges and nonmonotonic behavior in the case of purely NES effect.

Figure 3(d) shows, for the $\gamma=1$ case, the fraction of particles $N_{i} / N$ reaching within $t_{\max }$ the threshold position $x_{t}$ $=0.5$ into the potential well. This threshold position $x_{t}$ corresponds to the concavity change of the potential and is considered for this reason as a reference indicator for the effective entrance of the particle into the well. This ratio depends on the initial position, goes to zero at low noise intensities,
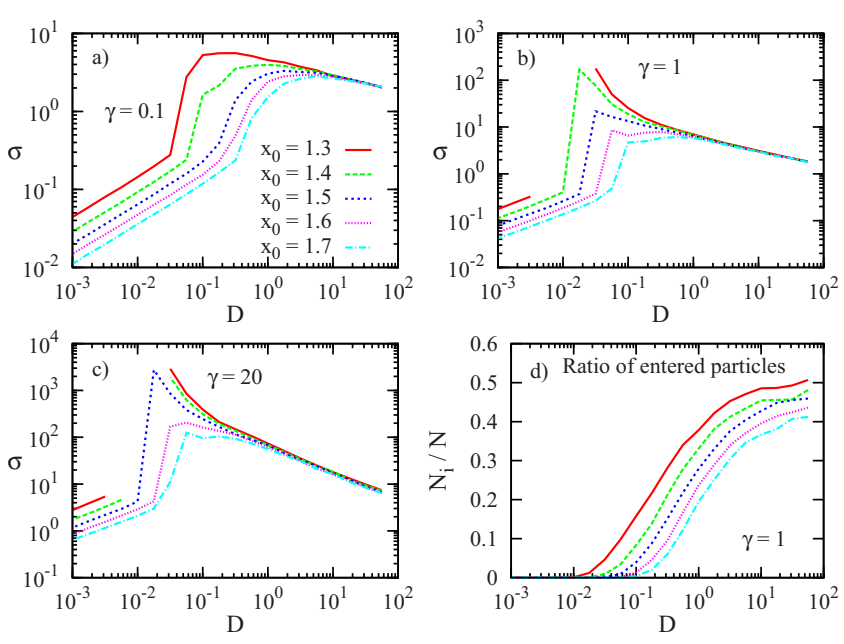

FIG. 3. (Color online) Log-log plot of the standard deviation $\sigma$ as a function of $D$ for $\gamma=0.1,1,20$ [panels (a)-(c), respectively] and the same initial positions in Fig. 1. Panel (d): semilogarithmic plot of the fraction of particle $N_{i} / N$ entering the potential well, within the maximum simulation time $t_{\max }$, as a function of the scaled noise intensity $D$.

and approaches the value of 0.5 at high enough noise intensity values.

Figure 4 shows more clearly the effect of decreasing the damping for a given initial position. The most important effect is the lowering of the maximum $\tau$ values. At high values of the noise intensity the mean first passage time decreases monotonously and the slope of this limit curve becomes flatter by decreasing the damping. This means that the lowering of the $\tau$ values in the NES region at intermediate noise values for decreasing damping is partially compensated by a larger noise intensity interval involved in the NES. The effect of the finite damping is therefore, on one hand, to reduce the NES in the escape process and the divergent behavior, on
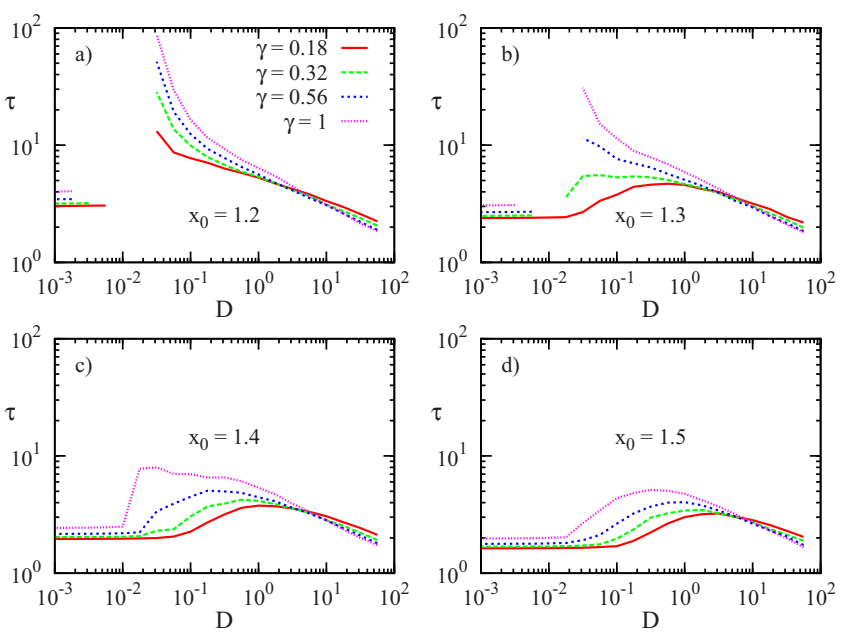

FIG. 4. (Color online) Log-log plot of the MFPT $\tau$ as a function of $D$ for fixed initial positions $\left(x_{0}=1.2,1.3,1.4,1.5\right)$ and for different values of the damping parameter, namely, $\gamma$ $=0.18,0.32,0.56,1$. We see how the divergent trend depends on $\gamma$ for a fixed $x_{0}$ value. This means that the critical position $x_{c}^{*}$ depends on the damping parameter $x_{c}^{*}=x_{c}^{*}(\gamma)$. 
the other hand, to shift the stability increase toward increasing values of the noise intensity.

We will focus now on the divergent behavior of the MFPT exhibited by the system. In the high damping regime, this divergence is observed for $x_{\max }<x_{0}<x_{c}$ and the curve is nonmonotonic for $x_{0} \geq x_{c}$, where $x_{\max }=1$ and $x_{c}=1.5$. By decreasing the value of $\gamma$ we observe [see Figs. 2(a) and 2(b)] a displacement of the maximum MFPT toward higher values of noise intensity and a correspondent shift of the critical initial position $x_{c}^{*}$ toward lower values. In fact, the initial position $x_{0}=1.4$ presents a clear divergent behavior for $D$ $\rightarrow 0$ in Fig. 2(c), while in Figs. 2(a) and 2(b) the curves for the same position show nonmonotonic behavior. Figure 2(a), where $\gamma=0.1$, shows NES humps for all the initial positions without any divergence.

We note that the quantity $x_{c}$ was theoretically defined for the overdamped metastable system in Refs. $[6,8,20]$. Here, $x_{c}^{*}$ is the same peculiar quantity, which discriminates between the initial positions giving divergence in the MFPT as $D$ $\rightarrow 0$ and those which give finite nonmonotonic behavior of MFPT as a function of $D$ in the underdamped system.

Because of no exact expression of MFPT available in the underdamped case, we solve the problem of finding $x_{c}^{*}$ by numerical simulations of the Langevin equation describing the transient dynamics of the metastable system. Specifically, we analyze the behavior of MFPT as a function of $D$, for fixed damping parameter $\gamma$ and different initial positions (Fig. 2) and for fixed initial position and different values of $\gamma$ (Fig. 4). We are able to find, within a good accuracy, the values of $x_{c}^{*}$ and $\gamma$ such that the curves corresponding to initial conditions within the range $x_{\max }<x_{0}<x_{c}^{*}$ have divergent behaviors. In particular, for instance, the average of the values corresponding to the two next curves of Fig. 4(b), which show different behaviors (divergent for $\gamma=0.56$ and not divergent for $\gamma=0.32$ ), gives the critical value of $\gamma_{c}$ $[0.44=(0.32+0.56) / 2$ in Fig. $4(\mathrm{~b})]$, corresponding to $x_{c}^{*}$ equal to the fixed initial condition considered $\left(x_{c}^{*}=x_{0}=1.3\right.$ in this example).

Our numerical simulations should be seen as numerical experiments and the results presented might depend, as in a real experiment, on the observation time and the number of samples. Since we present results for all the particles arriving the absorbing barrier within $t_{\max }$, both number could affect our results. However, the critical initial position $x_{c}^{*}$ does not depend on the observation time. What depends on the observation time is not the critical position but, instead, all the maximum values of MFPT of the curves showing divergent behavior. In other words, from inspection of Figs. 2 and 4 it is easy to recognize all the curves having divergent behavior from those with finite behavior. This peculiar scenario (divergent and not divergent curves) does not change because of $t_{\text {max }}$.

If we decrease the damping, at low noise intensities, the particles entering the potential well will escape more easily than in the overdamped case. This is due to the inertial term, which allows the particle to preserve the acquired energy for enough long time $(\sim 1 / \gamma)$ so that the escape will occur. Therefore, the trapping effect, which is responsible for the divergent behavior for any initial unstable state within the range $x_{\max }<x_{0}<x_{c}^{*}$, will happen with smaller and smaller

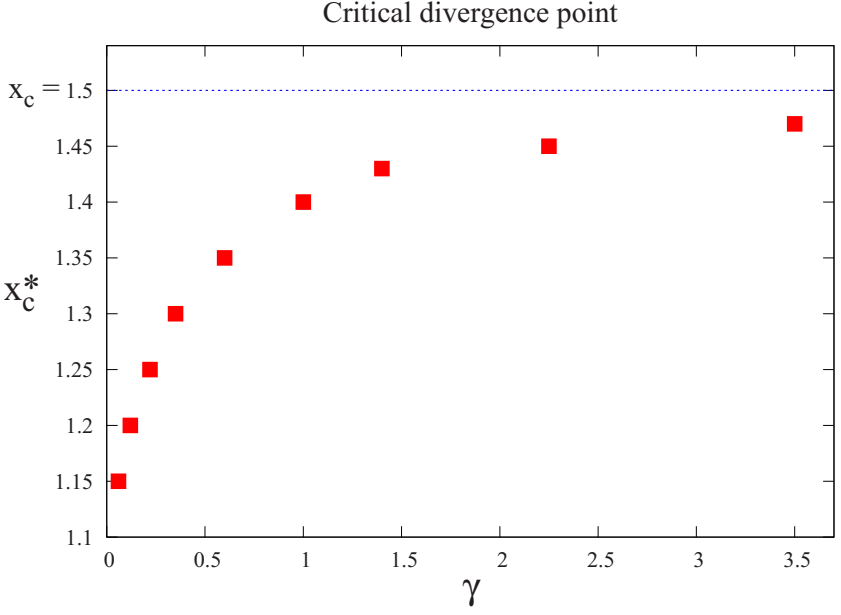

FIG. 5. (Color online) Critical initial position as a function of the damping parameter $\gamma$. By increasing the damping, $x_{c}^{*}$ tends asymptotically to the overdamped value $x_{c}=1.5$.

probability by decreasing the damping parameter [see, for instance, Fig. 2(a) $(\gamma=0.1)$ where no divergence is observed].

Figure 4 shows more clearly this trend. We note that the critical initial position becomes a function of the damping parameter $\left[x_{c}^{*}=x_{c}^{*}(\gamma)\right]$. In fact, in the different panels it is possible to note how the curves with different dampings show or not divergent behavior for low values of the noise intensity $D$. In particular, in Fig. 4(b) (initial position $x_{0}$ $=1.3$ ) we can see how the curves completely loose the divergent behavior by decreasing the value of the damping from $\gamma=1$ to $\gamma=0.18$.

The previous description can be summarized in Fig. 5 where it is evident that the critical initial position $x_{c}^{*}$ is an increasing saturating function of the damping parameter $\gamma$. For increasing values of $\gamma$, i.e., from low to high damping regime, $x_{c}^{*}$ tends asymptotically to the value $x_{c}=1.5$, which is the critical initial position in the overdamped dynamical regime. Our results have many similarities to those obtained for the case of an overdamped system in the presence of Gaussian colored noise [21]. These similarities could be ascribed to the fact that the correlation time in the overdamped regime (with colored noise) and the inertia in the underdamped regime (with white noise) play a similar memory effect in the dynamics of the system. In fact, the inverse of the damping parameter represents the time scale for energy conservation in the inertial dynamics. On the other hand, the correlation time of the colored noise fixes a time scale in which fluctuations preserve their features. The results here presented suggest that the two "preservation features" give similar behaviors in the MFPT from unstable initial position in a metastable system.

\section{CONCLUSIONS}

In this work we analyze the effect of the damping on the enhancement of stability phenomenon for a Brownian particle starting from unstable initial position and moving in a metastable system. We analyze different initial unstable po- 
sitions. We observe NES effect for all the initial positions investigated. We also see an increase of the NES range, i.e., the interval of the corresponding values of the noise intensity for which the effect is observed for decreasing values of the damping. In fact, the NES effect is always present when a particle is initially located beyond the local potential maximum in the escape region. The critical initial position, which discriminates between divergent and nonmonotonic behavior of MFPT at very low temperatures, is a function of the damping parameter. For small damping we find a purely nonmonotonic behavior of MFPT for all the initial unstable positions investigated and within the maximum simulation time. The range of the noise intensity values where the NES effect appears is shifted toward higher values for decreasing damping. By controlling the damping parameter, it is possible to find the dynamical regime where the NES phenomenon could be revealed in experiments of transient dynamics of metastable systems with damping.

Lastly, the peculiarities of the behavior of the MFPT appear to be similar to those observed for an overdamped Brownian particle in a metastable potential in the presence of colored noise.

\section{ACKNOWLEDGMENTS}

The authors acknowledge financial support from Spanish MICINN through Grant No. FIS2008-01240, cofinanced by FEDER funds, and the Italian MIUR.
[1] H. A. Kramers, Physica (Amsterdam) 7, 284 (1940).

[2] V. I. Mel'nikov, Phys. Rep. 209, 1 (1991); P. Hänggi, P. Talkner, and M. Borkovec, Rev. Mod. Phys. 62, 251 (1990).

[3] J. E. Hirsch, B. A. Huberman, and D. J. Scalapino, Phys. Rev. A 25, 519 (1982).

[4] I. Dayan, M. Gitterman, and G. H. Weiss, Phys. Rev. A 46, 757 (1992).

[5] R. N. Mantegna and B. Spagnolo, Phys. Rev. Lett. 76, 563 (1996).

[6] N. V. Agudov and B. Spagnolo, Phys. Rev. E 64, 035102(R) (2001).

[7] A. A. Dubkov, N. V. Agudov, and B. Spagnolo, Phys. Rev. E 69, 061103 (2004).

[8] A. Fiasconaro, D. Valenti, and B. Spagnolo, Physica A 325, 136 (2003)

[9] A. L. Pankratov and B. Spagnolo, Phys. Rev. Lett. 93, 177001 (2004).

[10] N. V. Agudov and A. N. Malakhov, Phys. Rev. E 60, 6333 (1999); F. Apostolico, L. Gammaitoni, F. Marchesoni, and S. Santucci, ibid. 55, 36 (1997); D. Dan, M. C. Mahato, and A. M. Jayannavar, ibid. 60, 6421 (1999); R. Wackerbauer, ibid. 59, 2872 (1999); A. Mielke, Phys. Rev. Lett. 84, 818 (2000); E. V. Pankratova, A. V. Polovinkin, and B. Spagnolo, Phys.
Lett. A 344, 43 (2005).

[11] A. Fiasconaro, B. Spagnolo, A. Ochab-Marcinek, and E. Gudowska-Nowak, Phys. Rev E 74, 041904 (2006).

[12] P. I. Hurtado, J. Marro, and P. L. Garrido, Phys. Rev. E 74, 050101(R) (2006).

[13] R. Mankin, E. Soika, A. Sauga, and A. Ainsaar, Phys. Rev. E 77, 051113 (2008).

[14] L. Ridolfi, P. D'Odorico, and F. Laio, J. Theor. Biol. 248, 301 (2007); P. D’Odorico, F. Laio, and L. Ridolfi, Proc. Natl. Acad. Sci. U.S.A. 102, 10819 (2005).

[15] J. I. A. Zheng-Lin, Chin. Phys. Lett. 25, 1209 (2008).

[16] M. Trapanese, J. Appl. Phys. 105, $07 \mathrm{D} 313$ (2009).

[17] J. H. Li, J. Phys.: Condens. Matter 22, 115702 (2010).

[18] M. Yoshimoto, H. Shirahama, and S. Kurosawa, J. Chem. Phys. 129, 014508 (2008).

[19] G. Sun, N. Dong, G. Mao, J. Chen, W. Xu, Z. Ji, L. Kang, P. Wu, Y. Yu, and D. Xing, Phys. Rev. E 75, 021107 (2007); C. Pan, X. Tan, Y. Yu, G. Sun, L. Kang, W. Xu, J. Chen, and P. Wu, ibid. 79, 030104(R) (2009).

[20] A. Fiasconaro, B. Spagnolo, and S. Boccaletti, Phys. Rev. E 72, 061110 (2005).

[21] A. Fiasconaro and B. Spagnolo, Phys. Rev. E 80, 041110 (2009). 